\title{
Association of medication adherence and depression with the control of low-density lipoprotein cholesterol and blood pressure in patients at high cardiovascular risk
}

This article was published in the following Dove Press journal:

Patient Preference and Adherence

Julius L Katzmann'

Felix Mahfoud ${ }^{2}$

Michael Böhm²

Martin Schulz ${ }^{3,4}$

Ulrich Laufs'

'Department of Cardiology, Universitätsklinikum Leipzig, Leipzig, Germany; ${ }^{2}$ Medical Clinic III, Cardiology, Angiology, Intensive Care, Universitätsklinikum des Saarlandes, Homburg, Germany; ${ }^{3}$ Department of Clinical Pharmacy and Biochemistry, Institute of Pharmacy, Freie Universität Berlin, Berlin, Germany; ${ }^{4}$ Department of Medicine, ABDA - Federal Union of German Associations of Pharmacists, Berlin, Germany
Correspondence: Julius L Katzmann Klinik und Poliklinik für Kardiologie, Universitätsklinikum Leipzig, Liebigstraße 20, 04103 Leipzig, Germany Tel +49 34I 97| 2650 Email julius.katzmann@medizin. uni-leipzig.de
Background: Many patients at high cardiovascular risk do not reach targets for low-density lipoprotein cholesterol (LDL-C) and blood pressure (BP). Depression is a frequent comorbidity in these patients and contributes to poor medication adherence.

Objective: The aim of this study was to elucidate the associations between adherence to lipidand BP-lowering drugs, the diagnosis of depression, and the control of LDL-C and BP.

Patients and methods: This study was conducted as multicenter, single-visit cross-sectional study in Germany. Adherence was assessed by the Morisky Medication Adherence Scale-8 (MMAS-8), and depression was assessed as documented in the patient chart.

Results: A total of 3,188 ambulatory patients with hypercholesterolemia (39.8\%), stable coronary artery disease (CAD; $7.4 \%)$, or both $(52.9 \%)$ were included. Patients had a history of myocardial infarction (30.8\%), diabetes $(42.0 \%)$, were smokers $(19.7 \%)$, and $16.1 \%$ had the investigatorreported diagnosis of depression. High or moderate adherence to lipid-lowering medication compared to low adherence was associated with lower LDL-C levels (105.5 \pm 38.3 vs $120.8 \pm 42.4$ $\mathrm{mg} / \mathrm{dL}$ ) and lower BP (systolic BP $133.4 \pm 14.5 \mathrm{vs} 137.9 \pm 13.9 \mathrm{mmHg}$, diastolic BP $78.3 \pm 9.6 \mathrm{vs}$ $81.8 \pm 9.6 \mathrm{mmHg}$ ) and with a higher proportion of patients achieving the guideline-recommended LDL-C ( $16.9 \%$ vs $10.1 \%$ ) and BP target ( $52.2 \%$ vs $40.8 \%$, all comparisons $P<0.0001$ ). Adherence was worse in patients with depression. Correspondingly, patients with depression showed higher LDL-C levels, higher BP, and a lower probability of achieving the LDL-C and BP goal. Medication adherence correlated between BP- and lipid-lowering medications.

Conclusion: Self-reported medication adherence can be easily obtained in daily practice. A low adherence and the diagnosis of depression identify patients at risk for uncontrolled LDL-C and BP who likely benefit from intensified care.

Keywords: adherence, blood pressure, cardiovascular disease, coronary artery disease, depression, LDL cholesterol, lipids, MMAS-8, prevention, statin

\section{Introduction}

Cardiovascular diseases (CVD) such as coronary artery disease (CAD) are the leading cause of death worldwide. ${ }^{1}$ Large randomized controlled trials have shown that lowering of low-density lipoprotein cholesterol (LDL-C) and blood pressure (BP) is beneficial in primary and secondary prevention of CVD. ${ }^{2,3}$

The current European Society of Cardiology (ESC)/European Atherosclerosis Society (EAS) guidelines recommend achieving LDL-C levels of $<70$ and $<100 \mathrm{mg} / \mathrm{dL}$ for patients at very high and high risk, respectively. ${ }^{4}$ However, despite the availability of 
effective and well-tolerated drugs, the LDL-C of the majority of patients remains uncontrolled..$^{5-7}$ There are several factors influencing the risk factor control including adherence to medication defined as "the extent to which patients take medications as prescribed by their health care providers". ${ }^{8}$ Adherence to lipid- and BP-lowering medication gets worse over time. A few months after initiation, a very significant part of the patients stops taking the prescribed medication. ${ }^{9-11}$ Poor statin adherence has been linked to cardiovascular and all-cause mortality. ${ }^{12-14}$ Adherence to statins is associated with improved LDL-C levels. ${ }^{15}$ Similarly, improving adherence to BP medication has been shown to improve BP control and clinical outcomes. ${ }^{16,17}$ However, it is not well studied whether the data regarding adherence to BP-lowering medication can be extrapolated to adherence to lipid-lowering medication. In general, poor adherence to cardiovascular medication causes a considerable proportion of cardiovascular events and deaths..$^{18,19}$

Up to $20 \%$ of patients with CVD suffer from major depression. ${ }^{20} \mathrm{~A}$ bidirectional relationship has been assumed between these two disease entities. ${ }^{21}$ Depression is likely to contribute to non-adherence to lipid- and BP-lowering drugs, which may represent a link between depressive symptoms and risk of CVD. ${ }^{22}$

Therefore, the aim of this study was to elucidate the association between adherence to lipid- and BP-lowering drugs and the diagnosis of depression.

\section{Patients and methods}

This multicenter, single-visit cross-sectional study was conducted in Germany between March 15, 2017, and September 15, 2017. General practitioners and specialists treating outpatients were encouraged to include 10 consecutive patients scheduled for routine appointment. Inclusion criteria were as follows:

- $\geq 18$ years of age

- Hypertension as documented diagnosis

- Hypercholesterolemia or stable CAD as documented diagnosis

- Current medication with at least one antihypertensive drug

- Current medication with a statin

- Signed informed consent.

Patients who had been hospitalized because of a cardiovascular event within the past 3 months were excluded.

After the patients had signed the informed consent, the participating physicians collected the following data:

- Age and sex

- Cardiovascular risk factors and comorbidities
- Depression (yes/no)

- Current antihypertensive medication

- Current statin medication

- Systolic and diastolic BP (SBP, DBP) values, measured at the documentation visit according to the ESC/European Society of Hypertension (ESH) guideline ${ }^{23}$

- Control of hypertension according to physician

- Serum lipid levels (total cholesterol, LDL-C, high-density lipoprotein cholesterol [HDL-C], triglycerides), if measured within the past 12 months

- Control of LDL-C according to physician

- Eligibility of the patient for taking a fixed-dose combination (FDC) according to the physician and the reason for prescribing FDC pills.

Adherence to antihypertensive and lipid-lowering medication was determined using the Morisky Medication Adherence Scale-8 (MMAS-8), a self-report questionnaire comprising eight items. ${ }^{24}$ MMAS- 8 values of $<6$ were classified as "low adherence", 6 or 7 as "moderate adherence", and 8 as "high adherence". Results regarding adherence are based on the MMAS-8 scores for adherence to lipid-lowering medication. LDL-C goals were defined according to the current ESC/EAS guideline as $<70 \mathrm{mg} / \mathrm{dL}$ in patients with $\mathrm{CAD}$ or at very high cardiovascular risk and $<100 \mathrm{mg} / \mathrm{dL}$ in patients at high cardiovascular risk. ${ }^{4}$

The study protocol was in accordance with the ethical guidelines of the 1975 Declaration of Helsinki and approved by the ethics committee (Ärztekammer des Saarlandes 307/16).

\section{Statistical analyses}

Categorical values are expressed as the percentage of the evaluable patients for each variable, excluding patients with missing data. Continuous data are expressed as mean \pm SD. Categorical data were compared using the chi-squared test; for continuous data, the two-sample $t$-test was used. For comparison of BP and LDL-C control according to ESC guideline vs investigator, the McNemar test was utilized. Logistic regression was used to identify factors associated with adherence, LDL-C control, and BP control. The following factors were included in the regression analyses: age (defined as males older than 55 years and females older than 65 years), sex, smoking, depression, history of myocardial infarction, diabetes, peripheral vascular disease, stroke or transient ischemic attack, chronic heart failure, renal disease, positive family history of CAD, positive family history of hypercholesterolemia, adherence to, respectively, lipid- and BP-lowering medication, single-pill combination treatment for hypertension, and single-pill combination treatment for 
hypercholesterolemia. The analyses were performed with SAS, version 9.4 (SAS Institute Inc., Cary, NC, USA). A $P$-value of $<0.05$ was considered statistically significant.

\section{Results}

A total of 1,247 physicians participated, who included 3,312 patients $(76.5 \%$ included by general practitioners, $23.5 \%$ by specialists), of whom 3,188 were available for final analysis.

\section{Demographic characteristics and risk factors}

The baseline characteristics are summarized in Table 1. Of the 3,188 patients, $60.9 \%$ were male, $61.4 \%$ were aged between 60 and 79 years. $39.8 \%$ of the patients had a diagnosis of hypercholesterolemia, 7.4\% had a diagnosis of stable CAD, and $52.9 \%$ had both diagnoses. $30.8 \%$ of the patients had a history of myocardial infarction. The most present cardiovascular risk factors were age (76.3\%, defined as males older than 55 years or females older than 65 years), diabetes (42.0\%), and positive family history of CAD (28.8\%); $19.7 \%$ of the patients were smoking.

\section{Patients with depression}

Depression was diagnosed in $16.1 \%$ of the patients $(n=512)$. The majority of patients with depression were female (57.4\%), corresponding to $23.9 \%$ of the female patients and $11.4 \%$ of the male patients were with depression $(P<0.0001)$. Among the patients with depression, a higher proportion had a history of previous stroke or transient ischemic attack (16.8\% vs $10.6 \%$ ), a positive family history of hypercholesterolemia (32.2\% vs $23.6 \%)$, chronic heart failure (26.4\% vs $17.6 \%)$, renal disease $(20.5 \%$ vs $13.8 \%)$, and familial hypercholesterolemia (34.0\% vs $24.0 \%$, for all comparisons $P<0.0001$ ). Patients with depression were more often smokers $(24.4 \%$ vs $18.8 \%, P=0.0032$ ) and were prescribed more pills per day $(7.2 \pm 3.3$ vs $6.2 \pm 3.2, P<0.0001)$. Adherence to lipid-lowering medication was worse in patients with depression (Table 1). In logistic regression analyses, depression was associated with poor BP control ( $P=0.0211)$, poor LDL-C control $(P=0.0452)$, and poor medication adherence $(P<0.0001)$.

\section{Patients with low vs moderate or high adherence to lipid-lowering medication}

$42.0 \%$ of the patients exhibited low, $28.1 \%$ moderate, and $29.9 \%$ high self-reported adherence to their lipid-lowering medication. There were no significant differences between patients with high vs moderate adherence in LDL-C levels as well as LDL-C and BP control. Therefore, patients with high and moderate adherence were analyzed as one group and compared to patients with low adherence (Figure 1).

In comparison to patients with moderate or high adherence, patients with low adherence were younger; a higher proportion had a family history of CAD (33.2\% vs $25.0 \%)$ and hypercholesterolemia (29.5\% vs $21.7 \%)$ and a history of depression (20.0\% vs $13.4 \%$, for all comparisons $P<0.0001$ ), peripheral vascular disease ( $13.3 \%$ vs $10.1 \%, P=0.0074)$, and familial hypercholesterolemia ( $28.9 \%$ vs $22.6 \%, P<0.0001)$. Patients with low adherence were significantly more often smokers $(27.1 \%$ vs $14.7 \%, P<0.0001)$. The number of daily doses was comparable in both groups $(6.3 \pm 3.0$ vs $6.5 \pm 3.3$, $P=0.2101)$. There was no correlation between the number of daily doses and adherence measured by MMAS-8 (Spearman's correlation coefficient $-0.00434, P=0.8118$ ). The data are summarized in Table 1.

High adherence was more common in women than in men (31.0\% vs $25.8 \%, P=0.0035)$. However, the proportion of low adherence was comparable in men and women $(41.7 \%$ vs $41.1 \%, P=0.7849)$.

An FDC treatment was thought to contribute to treatment goal attainment in $63.1 \%$ of all patients by improving adherence $(45.1 \%)$, patient convenience $(43.8 \%)$, cardiovascular protection (32.2\%), LDL-C control (30.1\%), BP control $(26.2 \%)$, and costs $(17.2 \%)$.

Logistic regression revealed that depression and smoking are strongly associated with poor adherence $(P<0.0001)$, independent of using the categorized MMAS- 8 or the score as continuous variable. In the model of MMAS- 8 score as continuous variable, additional but less strong associations with poor adherence were found for stroke $(P=0.0456)$, age (men older than 55 years or women older than 65 years, $P=0.0330)$, and positive family history of CAD $(P=0.0213)$ and hypercholesterolemia $(P=0.0041)$.

\section{Blood pressure}

The mean office SBP was $135.3 \pm 14.4 \mathrm{mmHg}$, and the mean DBP was $79.8 \pm 9.7 \mathrm{mmHg}$. BP control was achieved in $47.2 \%$ and $90.9 \%$ of the patients according to $\mathrm{ESC} / \mathrm{ESH}$ guideline on hypertension (target $<140 / 90 \mathrm{mmHg}$ ) and as assessed by the investigator, respectively $(P<0.0001)$.

In patients with depression, BP was significantly higher and BP control was achieved in a significantly lower proportion of patients (SBP 138.1 $\pm 15.6 \mathrm{vs}$ 134.8 $\pm 14.1 \mathrm{mmHg}$, DBP $81.8 \pm 10.4$ vs $79.4 \pm 9.6 \mathrm{mmHg}$, BP control according to ESC guideline $39.1 \%$ vs $48.7 \%$, for all comparisons $P<0.0001$, BP control according to investigator $87.7 \%$ vs $91.6 \%, P=0.0053$ ). Patients with low adherence 
Table I Demographic parameters, comorbidities, and medication adherence for the total population, for patients with vs without depression, and for patients with low vs moderate or high adherence

\begin{tabular}{|c|c|c|c|c|c|c|c|}
\hline & $\begin{array}{l}\text { Total } \\
n=3,188\end{array}$ & $\begin{array}{l}\text { Depression } \\
n=512\end{array}$ & $\begin{array}{l}\text { No depression } \\
n=2,676\end{array}$ & $\begin{array}{l}P \text {-value, } \\
\text { depression } \\
\text { vs no } \\
\text { depression }\end{array}$ & $\begin{array}{l}\text { Low } \\
\text { adherence } \\
\text { (MMAS-8, <6) } \\
n=1,273\end{array}$ & $\begin{array}{l}\text { Moderate } \\
\text { or high } \\
\text { adherence } \\
\text { (MMAS-8, } \\
6-8) n=1,757\end{array}$ & $\begin{array}{l}\text { P-value, } \\
\text { low vs } \\
\text { moderate } \\
\text { or high } \\
\text { adherence }\end{array}$ \\
\hline \multicolumn{8}{|l|}{ Age (years) } \\
\hline$<60$ & 22.3 & 20.2 & 22.7 & 0.23 & 26.2 & 19.6 & $<0.0001$ \\
\hline $60-64$ & 14.3 & 15.9 & 14.1 & 0.2754 & 16.0 & 13.0 & 0.0234 \\
\hline $65-74$ & 28.2 & 30.2 & 27.8 & 0.2742 & 26.9 & 29.5 & 0.1198 \\
\hline $75-79$ & 18.9 & 19.8 & 18.7 & 0.5439 & 16.4 & 20.4 & 0.0051 \\
\hline$\geq 80$ & 16.2 & 13.9 & 16.6 & 0.131 & 14.5 & 17.5 & 0.0315 \\
\hline \multicolumn{8}{|l|}{ Sex } \\
\hline Female & 39.1 & 57.4 & 35.5 & $<0.0001$ & 38.8 & 39.7 & 0.4889 \\
\hline Male & 60.9 & 42.6 & 64.5 & $<0.0001$ & 61.2 & 60.3 & 0.0432 \\
\hline \multicolumn{8}{|l|}{ Risk factors and comorbidities } \\
\hline Hypercholesterolemia & 39.8 & 36.7 & 39.6 & 0.2228 & 39.3 & 39.0 & 0.8564 \\
\hline Stable CAD & 7.4 & 6.6 & 7.4 & 0.5436 & 7.6 & 6.6 & $0.28 \mid 4$ \\
\hline $\begin{array}{l}\text { Hypercholesterolemia and } \\
\text { stable CAD }\end{array}$ & 52.9 & 54.5 & 51.6 & 0.2224 & 51.7 & 52.8 & 0.5491 \\
\hline History of myocardial infarction & 30.8 & 28.3 & 31.3 & 0.1771 & 31.7 & 30.8 & 0.6186 \\
\hline Stroke/transient ischemic attack & 11.6 & 16.8 & 10.6 & $<0.0001$ & 12.8 & 10.6 & 0.0598 \\
\hline Positive family history of CAD & 28.8 & 32.2 & 28.1 & 0.0596 & 33.2 & 25 & $<0.0001$ \\
\hline $\begin{array}{l}\text { Positive family history of } \\
\text { hypercholesterolemia }\end{array}$ & 25.0 & 32.2 & 23.6 & $<0.0001$ & 29.5 & 21.7 & $<0.0001$ \\
\hline Smoker & 19.7 & 24.4 & 18.8 & 0.0032 & 27.1 & 14.7 & $<0.0001$ \\
\hline Diabetes & 42.0 & 44.9 & 41.4 & 0.1371 & 43.0 & 41.0 & 0.2787 \\
\hline Chronic heart failure & 19.0 & 26.4 & 17.6 & $<0.0001$ & 20.1 & 17.8 & 0.1119 \\
\hline Depression & 16.1 & 100 & 0 & & 20.0 & 13.4 & $<0.0001$ \\
\hline Peripheral vascular disease & 11.6 & 13.7 & 11.2 & 0.1118 & 13.3 & 10.1 & 0.0074 \\
\hline Renal disease & 14.9 & 20.5 & 13.8 & $<0.0001$ & 14.5 & 15.0 & $0.70 \mid 4$ \\
\hline Familial hypercholesterolemia & 25.6 & 34.0 & 24.0 & $<0.0001$ & 28.9 & 22.6 & $<0.0001$ \\
\hline $\begin{array}{l}\text { Number of daily tablets } \\
\text { (mean } \pm \text { SD) }\end{array}$ & $6.4 \pm 3.2$ & $7.2 \pm 3.3$ & $6.2 \pm 3.2$ & $<0.0001$ & $6.3 \pm 3.0$ & $6.5 \pm 3.3$ & 0.2101 \\
\hline \multicolumn{8}{|l|}{ BP } \\
\hline $\mathrm{SBP}$ in $\mathrm{mmHg}($ mean $\pm \mathrm{SD})$ & $135.3 \pm 14.4$ & $138.1 \pm 15.7$ & $134.8 \pm \mid 4.1$ & $<0.0001$ & $137.9 \pm 13.9$ & $133.4 \pm 14.5$ & $<0.0001$ \\
\hline $\mathrm{DBP}$ in $\mathrm{mmHg}($ mean $\pm \mathrm{SD})$ & $79.8 \pm 9.7$ & $81.8 \pm 10.1$ & $79.4 \pm 9.6$ & $<0.0001$ & $81.8 \pm 9.6$ & $78.3 \pm 9.6$ & $<0.0001$ \\
\hline $\begin{array}{l}\text { BP control according to ESC } \\
\text { guideline }\end{array}$ & 47.2 & 39.1 & 48.7 & $<0.0001$ & 40.8 & 52.2 & $<0.0001$ \\
\hline \multicolumn{8}{|c|}{ MMAS-8 questionnaire, lipid-lowering medication } \\
\hline High adherence (score 8) & 29.9 & 21.8 & 31.4 & $<0.0001$ & & & \\
\hline $\begin{array}{l}\text { Moderate adherence } \\
\text { (score } 6 \text { or } 7 \text { ) }\end{array}$ & 28.1 & 26.3 & 28.5 & 0.3805 & & & \\
\hline Low adherence $($ score $<6$ ) & 42.0 & 51.8 & 40.1 & $<0.0001$ & & & \\
\hline \multicolumn{8}{|c|}{ MMAS-8 questionnaire, BP-lowering medication } \\
\hline High adherence (score 8) & 30.3 & 21.9 & 31.9 & $<0.0001$ & & & \\
\hline $\begin{array}{l}\text { Moderate adherence } \\
\text { (score } 6 \text { or } 7 \text { ) }\end{array}$ & 29.0 & 27.9 & 29.2 & 0.6681 & & & \\
\hline Low adherence (score <6) & 40.7 & 50.2 & 38.9 & $<0.0001$ & & & \\
\hline
\end{tabular}

Note: Values are in percentage if not stated otherwise.

Abbreviations: BP, blood pressure; CAD, coronary artery disease; DBP, diastolic BP; ESC, European Society of Cardiology; MMAS-8, Morisky Medication Adherence Scale-8; SBP, systolic BP. 
compared to those with moderate or high adherence had higher BP values and worse BP control according to ESC guideline and according to the treating physician's assessment (SBP $137.9 \pm 13.9$ vs $133.4 \pm 14.5 \mathrm{mmHg}$, DBP $81.8 \pm 9.6$ vs $78.3 \pm 9.6 \mathrm{mmHg}$, BP control according to ESC guideline $40.8 \%$ vs $52.2 \%$, BP control according to investigator $87.9 \%$ vs $93.4 \%$, for all comparisons $P<0.0001)$. BP was higher in women than in men $(136.2 \pm 14.28$ vs $134.9 \pm 14.24 \mathrm{mmHg}$, $P=0.0255)$, and BP control was achieved in $47.4 \%$ of men and $45.7 \%$ of women $(P=0.4032)$.
In logistic regression analyses, low adherence (MMAS-8 $<6$ ) was strongly associated with poor BP control, whereas a history of myocardial infarction was associated with better BP control $(P<0.0001)$. An association with poor BP control was also found for depression $(P=0.0211)$, renal disease $(P=0.0065)$, and FDC antihypertensive treatment $(P=0.0062)$.

The data are given in Table 1 and Figure 1A. The comparisons of SBP in the six subgroups with low, moderate, and high adherence with and without depression are depicted in

\section{A 55}
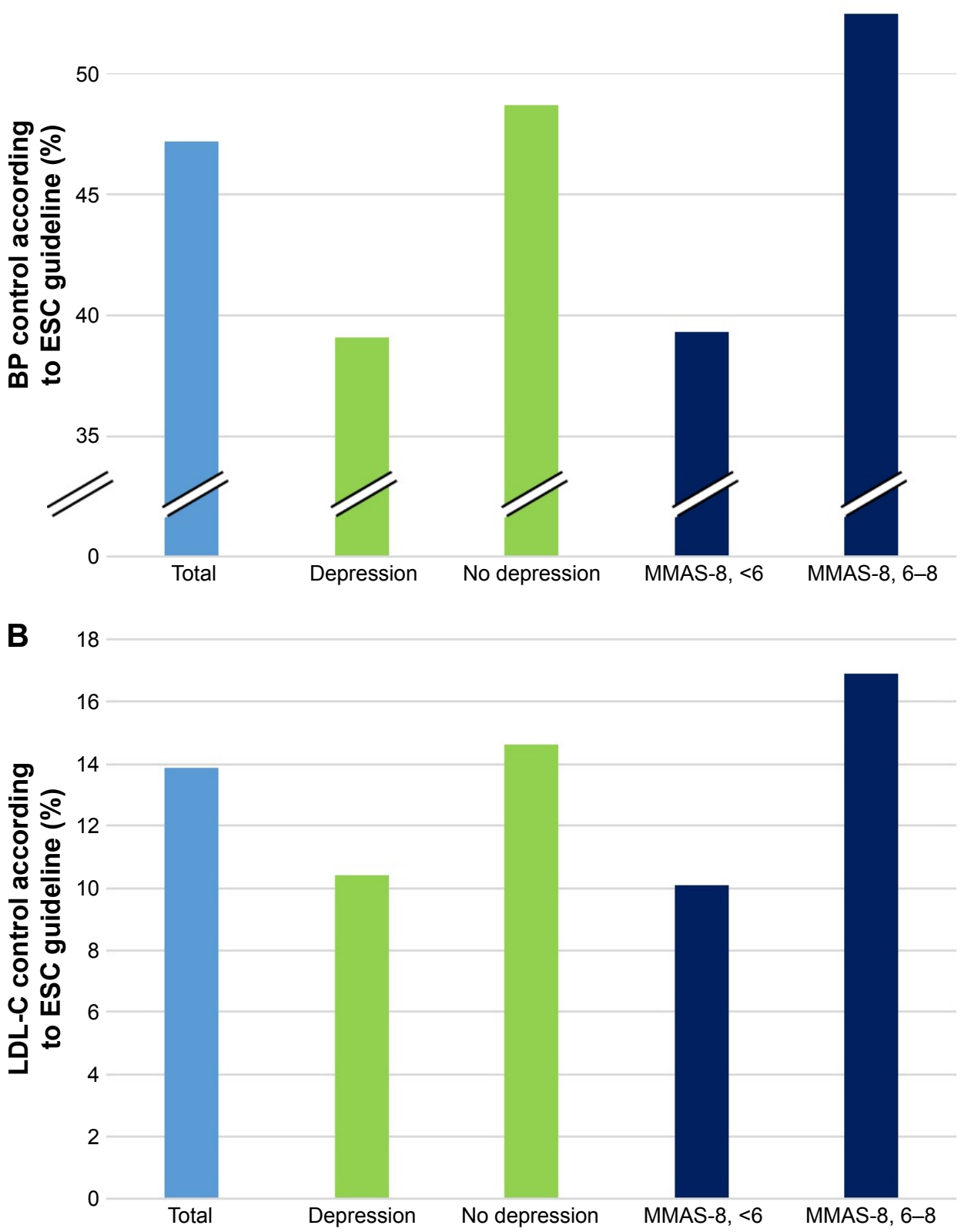

Figure I BP (A) and LDL-C control (B) in different subgroups.

Notes: BP (A) and LDL-C control (B) according to ESC guideline for the total population, patients with vs without depression and patients with low vs moderate or high adherence to BP- and lipid-lowering medication. MMAS-8, <6: low adherence; MMAS-8, 6-7: moderate adherence; MMAS-8, 8: high adherence.

Abbreviations: BP, blood pressure; ESC, European Society of Cardiology; LDL-C, low-density lipoprotein cholesterol; MMAS-8, Morisky Medication Adherence Scale-8. 

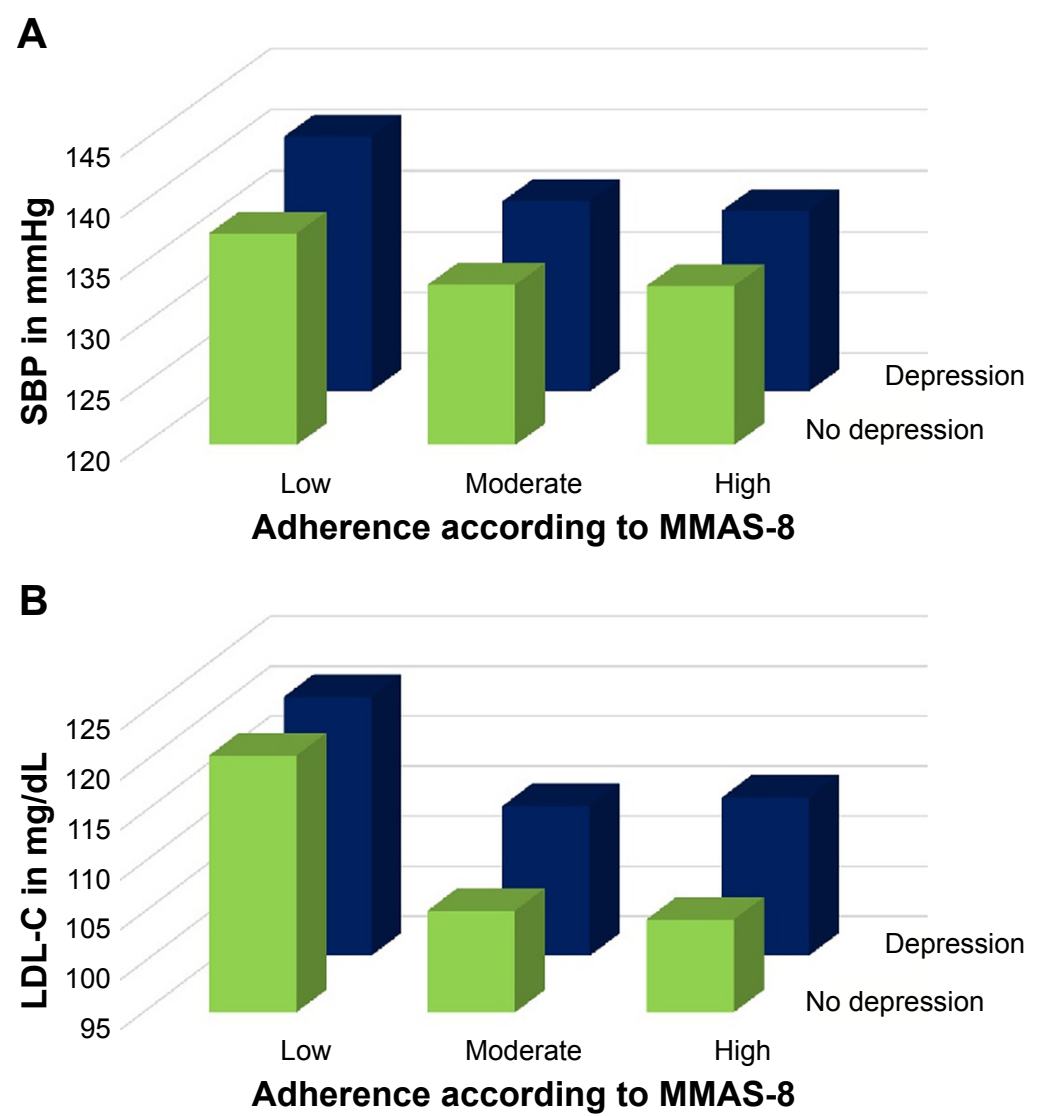

Figure 2 (A) SBP in patients with low, moderate, and high adherence with and without depression. (B) LDL-C levels in patients with low, moderate, and high adherence with and without depression.

Notes: (A) Low adherence: depression vs no depression: $P=0.0007$, moderate adherence: depression vs no depression: $P=0.0978$, high adherence: depression vs no depression: $P=0.2765$; depression: low vs moderate adherence: $P=0.0015$, moderate vs high adherence: $P=0.6904$, no depression: low vs moderate adherence: $P<0.0001$, moderate vs high adherence: $P=0.8798$. (B) Low adherence: depression vs no depression: $P=0.957 \mathrm{l}$, moderate adherence: depression vs no depression: $P=0.2460$, high adherence: depression vs no depression: $P=0.1827$; depression: low vs moderate adherence: $P=0.0224$, moderate vs high adherence: $P=0.8997$, no depression: low vs moderate adherence: $P<0.000$ I, moderate vs high adherence: $P=0.6721$. MMAS-8, $<6$ : low adherence; MMAS-8, 6-7: moderate adherence; MMAS-8, 8: high adherence.

Abbreviations: LDL-C, low-density lipoprotein cholesterol; MMAS-8, Morisky Medication Adherence Scale-8; SBP, systolic blood pressure.

Figure $2 \mathrm{~A}$ and indicate that SBP is related to both parameters. The results for DBP were comparable (not shown).

\section{Lipid-lowering medication, serum lipid levels, and LDL-C control}

Mean total cholesterol was $193.9 \pm 52.6 \mathrm{mg} / \mathrm{dL}$, LDL-C was $112.3 \pm 40.8 \mathrm{mg} / \mathrm{dL}$, HDL-C was $54.1 \pm 29.2 \mathrm{mg} / \mathrm{dL}$, and triglycerides were $148.6 \pm 93.7 \mathrm{mg} / \mathrm{dL}$. LDL-C control was achieved in $13.9 \%$ and $75.8 \%$ according to ESC guideline and investigator, respectively $(P<0.0001)$. The mean score of the MMAS-8 regarding lipid-lowering medication was 5.6 \pm 2.3 .

Patients with depression were characterized by significantly higher LDL-C levels compared to patients without depression ( $116.4 \pm 42.0 \mathrm{vs} 111.5 \pm 40.5 \mathrm{mg} / \mathrm{dL}, P=0.0238$ ) and had a lower probability of achieving LDL-C control according to ESC guideline ( $10.4 \%$ vs $14.6 \%, P=0.0114)$. LDL-C control as reported by the treating physician was not different between the groups $(78.4 \%$ vs $75.3 \%, P=0.1269)$. In addition, triglycerides were significantly higher in patients with depression ( $161.2 \pm 93.8$ vs $146.1 \pm 93.5 \mathrm{mg} / \mathrm{dL}, P=0.0032)$. The proportion of patients taking statins, ezetimibe, or other lipid-lowering medication was comparable. Patients with low adherence to lipid-lowering medication showed significantly higher LDL-C levels compared to patients with moderate or high adherence $(120.8 \pm 42.4$ vs $105.5 \pm 38.3 \mathrm{mg} / \mathrm{dL}$, $P<0.0001)$. A smaller proportion of patients with low adherence achieved LDL-C control (LDL-C control according to ESC guideline $10.1 \%$ vs $16.9 \% P<0.0001$, LDL-C control according to investigator $72.4 \%$ vs $78.4 \%, P=0.0001$ ). Patients with low adherence had higher levels of triglycerides (162.8 \pm 94.4 vs $136.6 \pm 90.8, P<0.0001)$. The proportion of patients taking statins, ezetimibe, or other lipid-lowering medication was comparable. Women had higher LDL-C levels than men $(120.3 \pm 43.6$ vs $109.1 \pm 38.86 \mathrm{mg} / \mathrm{dL}$, $P<0.0001$ ), whereas in men triglycerides were higher 
Table 2 Serum lipid levels, LDL-C control, and lipid-lowering medication for the total population, patients with vs without depression, and patients with low vs moderate or high adherence to lipid-lowering medication

\begin{tabular}{|c|c|c|c|c|c|c|c|}
\hline & $\begin{array}{l}\text { Total } \\
\mathbf{N}=3,188\end{array}$ & $\begin{array}{l}\text { Depression } \\
n=512\end{array}$ & $\begin{array}{l}\text { No } \\
\text { depression } \\
n=2,676\end{array}$ & $\begin{array}{l}P \text {-value, } \\
\text { depression } \\
\text { vs no } \\
\text { depression }\end{array}$ & $\begin{array}{l}\text { Low } \\
\text { adherence } \\
\text { (MMAS-8, } \\
\text { <6) n=l,273 }\end{array}$ & $\begin{array}{l}\text { Moderate or } \\
\text { high adherence } \\
\text { (MMAS-8, 6-8) } \\
n=1,757\end{array}$ & $\begin{array}{l}\text { P-value low } \\
\text { vs moderate } \\
\text { or high } \\
\text { adherence }\end{array}$ \\
\hline Total cholesterol & $193.9 \pm 52.6$ & $203.5 \pm 52.4$ & $192.0 \pm 52.5$ & $<0.0001$ & $206.4 \pm 52.9$ & $184.5 \pm 51.0$ & $<0.0001$ \\
\hline LDL-C & $112.3 \pm 40.8$ & $116.4 \pm 42.0$ & $111.5 \pm 40.5$ & 0.0238 & $120.8 \pm 42.4$ & $105.5 \pm 38.3$ & $<0.0001$ \\
\hline Triglycerides & $148.6 \pm 93.7$ & $161.2 \pm 93.8$ & $146.1 \pm 93.5$ & 0.0032 & $162.8 \pm 94.4$ & $136.6 \pm 90.8$ & $<0.0001$ \\
\hline HDL-C & $51.1 \pm 29.2$ & $53.3 \pm 20.3$ & $54.3 \pm 30.7$ & 0.4495 & $53.8 \pm 31.9$ & $53.7 \pm 21.0$ & 0.9248 \\
\hline $\begin{array}{l}\text { LDL-C controlled according } \\
\text { to investigator }(\%)\end{array}$ & 75.8 & 78.4 & 75.3 & 0.1269 & 72.4 & 78.4 & 0.0001 \\
\hline $\begin{array}{l}\text { LDL-C controlled according } \\
\text { to ESC guideline (\%) }\end{array}$ & 13.9 & 10.4 & 14.6 & 0.0114 & 10.1 & 16.9 & $<0.0001$ \\
\hline \multicolumn{8}{|l|}{ Lipid-lowering medication } \\
\hline Statin (\%) & 80.8 & 78.1 & 81.3 & 0.3677 & 81.1 & 80.9 & 0.2407 \\
\hline Statin/ezetimibe FDC (\%) & 12.1 & 13.9 & 11.7 & 0.1233 & 11.0 & 12.8 & 0.219 \\
\hline Others (\%) & 4.5 & 4.4 & 4.6 & 0.9092 & 4.7 & 4.1 & 0.3812 \\
\hline Ezetimibe (\%) & 2.6 & 3.6 & 2.4 & 0.1121 & 3.1 & 2.2 & 0.0961 \\
\hline
\end{tabular}

Note: Values are in milligram per deciliter and presented as mean \pm SD if not stated otherwise.

Abbreviations: ESC, European Society of Cardiology; FDC, fixed-dose combination; HDL-C, high-density lipoprotein cholesterol; LDL-C, low-density lipoprotein cholesterol; MMAS-8, Morisky Medication Adherence Scale-8.

$(152.9 \pm 97.15$ vs $142 \pm 86.7 \mathrm{mg} / \mathrm{dL}, P=0.0081)$ and HDL-C was lower ( $51.4 \pm 28.57$ vs $57.9 \pm 24.45 \mathrm{mg} / \mathrm{dL}, P<0.0001)$. LDL-C control according to guideline was documented in $15.5 \%$ of men and $10.8 \%$ of women $(P=0.0006)$.

Logistic regression revealed that male sex $(P=0.0006)$, stroke $(P=0.0014)$, and statin/ezetimibe single-pill combination treatment $(P=0.0005)$ were associated with better LDL-C control, whereas depression $(P=0.0452)$, renal disease $(P=0.0071)$, and low adherence (MMAS- $8<6, P=0.0015)$ were associated with worse LDL-C control. The data are given in Table 2 and Figure 1B.

The comparisons of LDL-C levels in the six subgroups with low, moderate, and high adherence with and without depression are depicted in Figure $2 \mathrm{~B}$ and indicate that serum LDL-C levels are related to both parameters.

\section{Comparison of adherence to BP- vs lipid-lowering medication}

The results of the MMAS- 8 scores for BP-and lipid-lowering medication were compared and showed a significant correlation (Figure 3, Spearman's correlation coefficient 0.92059, $P<0.0001)$.

\section{Discussion}

This study reports three important findings. The data demonstrate that low self-reported medication adherence is strongly associated with insufficient LDL-C and BP control.
The information on medication adherence can be reliably obtained in daily practice using the MMAS-8 questionnaire. Second, the clinical diagnosis of depression is associated with low LDL-C and BP target attainment. Low adherence and depression identify individuals among the cardiovascular high-risk population who might benefit from intensified care. Third, adherence to lipid-lowering medication correlates with adherence to BP-lowering medication. This information extends previous data from the literature ${ }^{10,11,16,17,25}$ on medication adherence to the situation in lipid lowering, which is closely associated with BP control.

Control of LDL-C as recommended by the current ESC/ EAS guideline ${ }^{4}$ was achieved in only $13.9 \%$ of the patients in this study. The poor LDL-C control is in line with previous reports of LDL-C goal attainment in USA and in Europe, which ranges between $20 \%$ and 30\%. ${ }^{5,7,26-28}$ As shown recently, potentially $73.9 \%$ of all patients at very high risk are able to reach the LDL-C goal of $<70 \mathrm{mg} / \mathrm{dL}$ if high-dose statins and ezetimibe are used at appropriate doses. ${ }^{29}$ Similarly, the ESC/ESH BP targets are achieved in only 50\%-60\% of the patients in USA and in Europe. ${ }^{7,23,30,31}$

Several reasons for the underutilization of well-proven therapies in daily practice have been discussed. One frequent reason for not prescribing or up-titrating statins is worries about potential side effects, although observed side effects occur infrequently and have been shown not to outweigh the beneficial effects. ${ }^{32-34}$ In addition, our data show a lack 


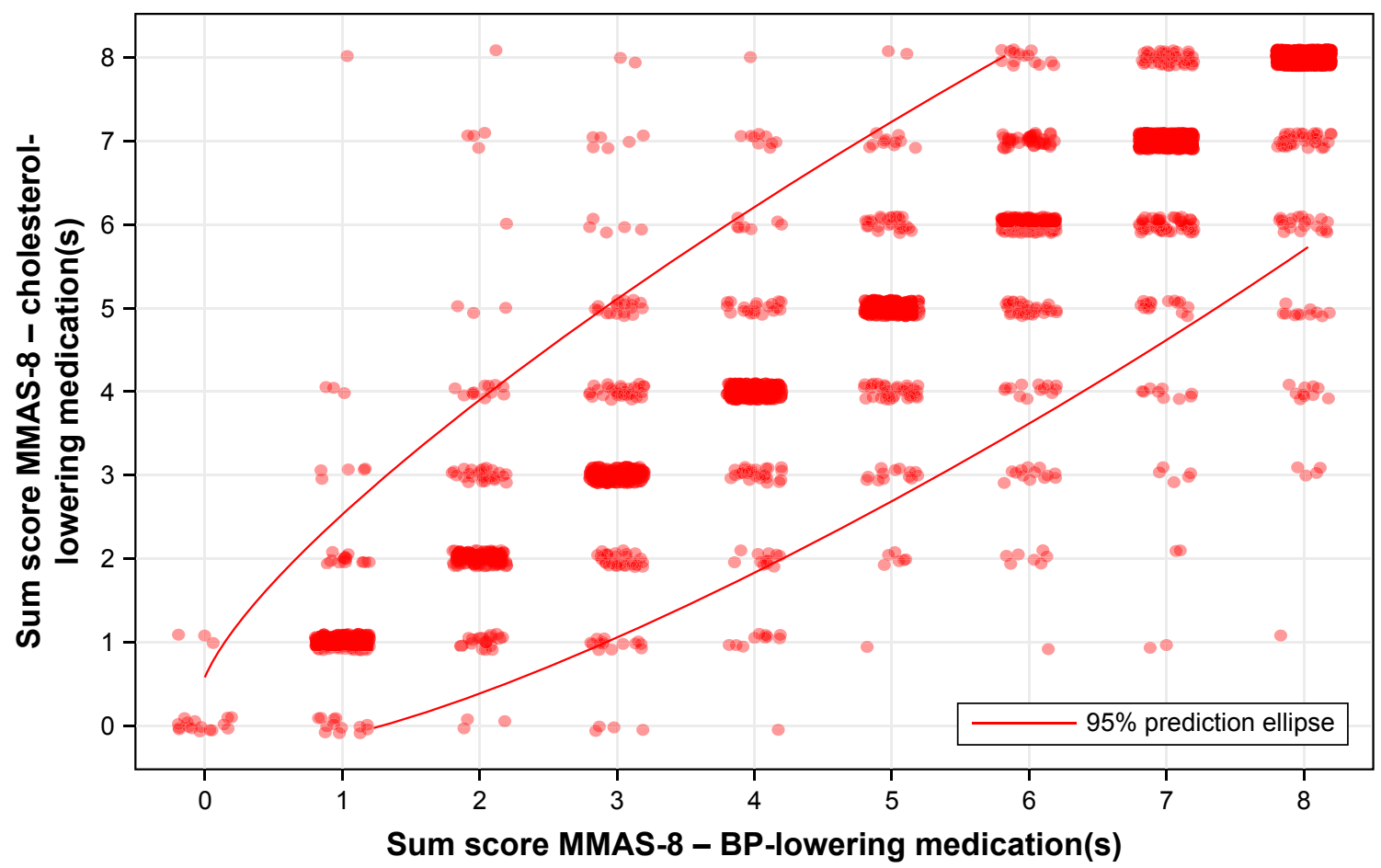

Figure 3 Correlation of MMAS-8 results for lipid- and BP-lowering medication.

Note: Spearman's correlation coefficient $0.92059, P<0.000$ I.

Abbreviations: BP, blood pressure; MMAS-8, Morisky Medication Adherence Scale-8.

of awareness regarding the guideline-recommended target values, reflected in our study by the treating physician's self-reported assessment of LDL-C control being achieved in $75.8 \%$ of the patients. Similarly, BP control was thought to be achieved in $90.9 \%$ of the patients when judged by treating physician as compared to $47.2 \%$ according to the current ESC/ESH guideline. The discrepancy in BP control between guideline recommendation and physicians' assessment is not of the same magnitude compared to the assessment of LDL-C control, indicating a different grade of awareness and implementation of the guideline recommendations. This is further underpinned by the fact that poor BP control was much stronger associated with poor medication adherence as patient-related factor than poor LDL-C control was, indicating that physicians' inertia is one main factor in poor LDL-C control. This interesting and novel finding indicates an important opportunity for future educational activities.

In light of revised BP targets, the number of patients with controlled hypertension may become even worse. ${ }^{35,36}$ It is indeed essential to stress the importance of BP lowering to reduce major cardiovascular end points and mortality. Based on a meta-analysis including more than 600,000 patients, an office BP reduction of $10 \mathrm{mmHg}$ is associated with an RR reduction in CAD by $17 \%$, stroke by $27 \%$, heart failure by $27 \%$, and mortality by $13 \%$, respectively. ${ }^{37}$
There are different methods for assessing adherence such as electronic measures, pharmacy refills, plasma/urine drug and/or metabolite concentrations, pill counts, and selfreport. ${ }^{38}$ Importantly, differences in the estimated adherence depend on the method used. ${ }^{39}$ The MMAS-8 self-reported questionnaire has the advantage of being relatively quickly to answer, easily applicable, and low cost for use in daily practice. $^{40,41}$

In our study, patients with low adherence to lipid-lowering medication were younger than those with moderate or high adherence. This is in line with studies in patients with chronic heart failure and hypertension. ${ }^{25,42}$ Furthermore, patients with low adherence had more CVD risk factors such as smoking, hypercholesterolemia, peripheral vascular disease, and positive family history of CAD. Therefore, these young patients are at high risk for the development and progression of CVD with a high potential for improvements in primary and secondary prevention by improved adherence. The abovementioned findings support the concept of the "healthy adherer effect", meaning that high medication adherence is a surrogate marker for overall healthy behavior. ${ }^{43,44}$

Our results confirm that low adherence correlates with fewer patients reaching LDL-C control. ${ }^{45}$ In our study, the rates of goal attainment did not differ significantly between moderate and high adherence, indicating that there is a 
certain extent of adherence which should not be undercut to reach a treatment goal. A similar result was reported by Chi et al. ${ }^{15}$ A possible explanation is that LDL-C lowering can still occur when doses are missed by patients with moderate adherence.

Low adherence to lipid-lowering medication not only did result in worse LDL-C control but also was associated with insufficient BP control in patients treated with antihypertensive drugs, further increasing cardiovascular risk. Our study suggests that adherence to BP-lowering medication may predict the intake of lipid-lowering medication. This finding has very important practical implications. Although several studies have proved that FDCs reduce BP to a greater extent compared to the components given separately, ${ }^{46-48}$ this type of information is missing for lipid-lowering medications, eg, statin-ezetimibe combinations. The observed robust correlation of the MMAS-8 questionnaires for lipid- and BP-lowering medication implies that the data on the potential benefit of FDCs may be extrapolated from BP to lipid lowering.

Another important finding of our study is that patients with past or present depression are less likely to achieve the guideline-recommended LDL-C goal. ${ }^{22,49}$ Patients with depression had more risk factors for CVD such as smoking or past stroke and are therefore at even higher risk for progressive CVD. Noteworthy, the treating physician's assessment of LDL-C control did not differ between patients with vs without depression in contrast to the goal attainment according to guideline, which was significantly worse in patients with depression. One might suggest that the symptoms attributable to depression may have been dominant in the patients' treatment, potentially distracting from somatic problems. Patients with depression may benefit from the treatment of depression by an improvement in medication adherence. ${ }^{50}$

Our study shows a higher prevalence of depression in women (23.9\% vs $11.4 \%$ in men). Although depression was strongly associated with adherence, sex was no predictor of poor adherence. This is further underpinned by a comparable proportion of men and women with achieved BP control, which was strongly associated with adherence. LDL-C control was worse in women. Poor LDL-C control in women seems to be driven by depression rather than poor adherence, suggesting that especially in women LDL-C is an underappreciated risk factor prone to physicians' inertia.

\section{Limitations}

Our study has some limitations. The cross-sectional study design carries limited possibilities for exploring causal relationships and does not provide clinical follow-up.
The consecutive inclusion of the patients was not supervised; therefore, selection bias was possible. Depression was not diagnosed using special questionnaires or diagnostic criteria, but was assessed according to the treating physician's diagnosis; however, this reflects the situation in daily practice. Similarly, the self-reported MMAS- 8 is less precise and overestimates adherence compared to electronic monitoring, pharmacy claims, or refill data or by measuring drug/ metabolite levels in the blood and/or urine, ${ }^{38}$ however, the advantage of the method is the feasibility and practicality in daily practice that we documented in our study.

\section{Conclusion}

The majority of patients at high cardiovascular risk did not reach the guideline-recommended LDL-C und BP goals. Low adherence and the diagnosis of depression identified individuals at risk for reduced LDL-C and BP control who are likely to benefit from intensified care. Self-reported medication adherence can be easily obtained in daily practice.

\section{Acknowledgments}

The study was funded by Servier Deutschland GmbH, Germany. Statistical analyses were supported by Pharmalog, Institut für klinische Forschung $\mathrm{GmbH}$, Oskar-MessterStraße 29, Ismaning, Germany.

\section{Author contributions}

JLK and UL designed the study and wrote the article. All authors contributed to data analysis, drafting or revising the article, gave final approval of the version to be published, and agree to be accountable for all aspects of the work.

\section{Disclosure}

Julius L Katzmann reports grants from Servier Deutschland $\mathrm{GmbH}$, Germany, non-financial support from Pharmalog, Institut für klinische Forschung $\mathrm{GmbH}$, Oskar-MessterStraße 29, Ismaning, Germany, during the conduct of the study; Michael Böhm reports personal fees from Amgen, personal fees from Bayer, personal fees from Servier, personal fees from Medtronic, personal fees from Boehringer Ingelheim, nothing from Vifor, personal fees from Bristol Myers Squibb, outside the submitted work; and Ulrich Laufs report other from Servier, during the conduct of the study. The authors report no other conflicts of interest in this work.

\section{References}

1. World Health Organization [homepage on the Internet]. Global Health Observatory (GHO) data. Available from: http://www.who.int/gho/ncd/ mortality_morbidity/en/. Accessed May 21, 2018. 
2. Collins R, Reith C, Emberson J, et al. Interpretation of the evidence for the efficacy and safety of statin therapy. Lancet. 2016;388(10059): 2532-2561.

3. Baigent C, Keech A, Kearney PM, et al; Cholesterol Treatment Trialists' (CTT) Collaborators. Efficacy and safety of cholesterol-lowering treatment: prospective meta-analysis of data from 90,056 participants in 14 randomised trials of statins. Lancet. 2005;366(9493):1267-1278.

4. Catapano AL, Graham I, de Backer G, et al; ESC Scientific Document Group. 2016 ESC/EAS Guidelines for the Management of Dyslipidaemias. Eur Heart J. 2016;37(39):2999-3058.

5. Chiang CE, Ferrières J, Gotcheva NN, et al. Suboptimal Control of Lipid Levels: Results from 29 Countries Participating in the Centralized Pan-Regional Surveys on the Undertreatment of Hypercholesterolaemia (CEPHEUS). $J$ Atheroscler Thromb. 2016;23(5):567-587.

6. Gitt AK, Lautsch D, Ferrieres J, et al. Low-density lipoprotein cholesterol in a global cohort of 57,885 statin-treated patients. Atherosclerosis. 2016;255:200-209.

7. Kotseva K, Wood D, De Bacquer D, et al; EUROASPIRE Investigators. EUROASPIRE IV: A European Society of Cardiology survey on the lifestyle, risk factor and therapeutic management of coronary patients from 24 European countries. Eur J Prev Cardiol. 2016;23(6):636-648.

8. Osterberg L, Blaschke T. Adherence to medication. N Engl J Med. 2005; 353(5):487-497.

9. Egan BM, Li J, Qanungo S, Wolfman TE. Blood pressure and cholesterol control in hypertensive hypercholesterolemic patients: national health and nutrition examination surveys 1988-2010. Circulation. 2013; 128(1):29-41.

10. Ewen S, Meyer MR, Cremers B, et al. Blood pressure reductions following catheter-based renal denervation are not related to improvements in adherence to antihypertensive drugs measured by urine/plasma toxicological analysis. Clin Res Cardiol. 2015;104(12):1097-1105.

11. Schulz M, Krueger K, Schuessel K, et al. Medication adherence and persistence according to different antihypertensive drug classes: A retrospective cohort study of 255,500 patients. Int J Cardiol. 2016; 220:668-676.

12. Kurlansky P, Herbert M, Prince S, Mack M. Coronary Artery Bypass Graft Versus Percutaneous Coronary Intervention: Meds Matter: Impact of Adherence to Medical Therapy on Comparative Outcomes. Circulation. 2016;134(17):1238-1246.

13. Daskalopoulou SS, Delaney JA, Filion KB, Brophy JM, Mayo NE, Suissa S. Discontinuation of statin therapy following an acute myocardial infarction: a population-based study. Eur Heart J. 2008;29(17): 2083-2091.

14. De Vera MA, Bhole V, Burns LC, Lacaille D. Impact of statin adherence on cardiovascular disease and mortality outcomes: a systematic review. Br J Clin Pharmacol. 2014;78(4):684-698.

15. Chi MD, Vansomphone SS, Liu IL, et al. Adherence to statins and LDL-cholesterol goal attainment. Am J Manag Care. 2014;20(4): e105-e112.

16. Corrao G, Parodi A, Nicotra F, et al. Better compliance to antihypertensive medications reduces cardiovascular risk. J Hypertens. 2011; 29(3):610-618.

17. Mazzaglia G, Ambrosioni E, Alacqua M, et al. Adherence to antihypertensive medications and cardiovascular morbidity among newly diagnosed hypertensive patients. Circulation. 2009;120(16):1598-1605.

18. Chowdhury R, Khan H, Heydon E, et al. Adherence to cardiovascular therapy: a meta-analysis of prevalence and clinical consequences. Eur Heart J. 2013;34(38):2940-2948.

19. Böhm M, Schumacher H, Laufs U, et al. Effects of nonpersistence with medication on outcomes in high-risk patients with cardiovascular disease. Am Heart J. 2013;166(2):306.e1-314.e1.

20. Sheps DS, Sheffield D. Depression, anxiety, and the cardiovascular system: the cardiologist's perspective. J Clin Psychiatry. 2001;62(Suppl 8): 12-6; discussion 17-8.

21. Khawaja IS, Westermeyer JJ, Gajwani P, Feinstein RE. Depression and coronary artery disease: the association, mechanisms, and therapeutic implications. Psychiatry (Edgmont). 2009;6(1):38-51.
22. DiMatteo MR, Lepper HS, Croghan TW. Depression is a risk factor for noncompliance with medical treatment: meta-analysis of the effects of anxiety and depression on patient adherence. Arch Intern Med. 2000; 160(14):2101-2107.

23. Mancia G, Fagard R, Narkiewicz K. 2013 ESH/ESC guidelines for the management of arterial hypertension: the Task Force for the Management of Arterial Hypertension of the European Society of Hypertension $(\mathrm{ESH})$ and of the European Society of Cardiology (ESC). Eur Heart J. 2013;34(28):2159-2219.

24. Morisky DE, Ang A, Krousel-Wood M, Ward HJ. Predictive validity of a medication adherence measure in an outpatient setting. J Clin Hypertens (Greenwich). 2008;10(5):348-354.

25. Gupta P, Patel P, Štrauch B, et al. Biochemical Screening for Nonadherence Is Associated With Blood Pressure Reduction and Improvement in Adherence. Hypertension. 2017;70(5):1042-1048.

26. Laufs U, Karmann B, Pittrow D. Atorvastatin treatment and LDL cholesterol target attainment in patients at very high cardiovascular risk. Clin Res Cardiol. 2016;105(9):783-790.

27. Fox KM, Tai MH, Kostev K, Hatz M, Qian Y, Laufs U. Treatment patterns and low-density lipoprotein cholesterol (LDL-C) goal attainment among patients receiving high- or moderate-intensity statins. Clin Res Cardiol. 2018;107(5):380-388.

28. Jones PH, Nair R, Thakker KM. Prevalence of dyslipidemia and lipid goal attainment in statin-treated subjects from 3 data sources: a retrospective analysis. J Am Heart Assoc. 2012;1(6):e001800.

29. Gitt AK, Lautsch D, De Ferrari G, Horack M, Brudi P, Ferriéres J. Consequent use of available oral lipid Lowering agents would bring the majority of high-risk patients with coronary heart disease to recommended targets: an estimate based on the DYSIS II Study. Clin Res Cardiol. 2018;107(Suppl 1).

30. Neuhauser HK, Adler C, Rosario AS, Diederichs C, Ellert U. Hypertension prevalence, awareness, treatment and control in Germany 1998 and 2008-11. J Hum Hypertens. 2015;29(4):247-253.

31. Joffres M, Falaschetti E, Gillespie C, et al. Hypertension prevalence, awareness, treatment and control in national surveys from England, the USA and Canada, and correlation with stroke and ischaemic heart disease mortality: a cross-sectional study. BMJ Open. 2013;3(8):e003423.

32. Mach F, Ray KK, Wiklund O, et al; European Atherosclerosis Society Consensus Panel. Adverse effects of statin therapy: perception vs. the evidence - focus on glucose homeostasis, cognitive, renal and hepatic function, haemorrhagic stroke and cataract. Eur Heart $J$. 2018;39(27):2526-2539.

33. Spence JD, Dresser GK. Overcoming challenges with statin therapy. J Am Heart Assoc. 2016;5(1):e002497.

34. Zhang H, Plutzky J, Shubina M, Turchin A. Continued Statin Prescriptions After Adverse Reactions and Patient Outcomes: A Cohort Study. Ann Intern Med. 2017;167(4):221-227.

35. Whelton PK, Carey RM, Aronow WS, et al. 2017 ACC/AHA/AAPA/ ABC/ACPM/AGS/APhA/ASH/ASPC/NMA/PCNA Guideline for the Prevention, Detection, Evaluation, and Management of High Blood Pressure in Adults: A Report of the American College of Cardiology/ American Heart Association Task Force on Clinical Practice Guidelines. $J$ Am Coll Cardiol. 2018;71(19):e127-e248.

36. Williams B, Mancia G, Spiering W, et al; ESC Scientific Document Group. 2018 ESC/ESH Guidelines for the management of arterial hypertension. Eur Heart J. 2018;39(33):3021-3104.

37. Ettehad D, Emdin CA, Kiran A, et al. Blood pressure lowering for prevention of cardiovascular disease and death: a systematic review and meta-analysis. Lancet. 2016;387(10022):957-967.

38. Jeffery RA, Navarro T, Wilczynski NL, et al. Adherence measurement and patient recruitment methods are poor in intervention trials to improve patient adherence. J Clin Epidemiol. 2014;67(10):1076-1082.

39. Morris AB, Li J, Kroenke K, Bruner-England TE, Young JM, Murray MD. Factors associated with drug adherence and blood pressure control in patients with hypertension. Pharmacotherapy. 2006;26(4): 483-492.

40. Lehmann A, Aslani P, Ahmed R, et al. Assessing medication adherence: options to consider. Int J Clin Pharm. 2014;36(1):55-69. 
41. Hawkshead J, Krousel-Wood MA. Techniques for Measuring Medication Adherence in Hypertensive Patients in Outpatient Settings. Dis Manag Health Outcomes. 2007;15(2):109-118.

42. Krueger K, Botermann L, Schorr SG, Griese-Mammen N, Laufs U, Schulz M. Age-related medication adherence in patients with chronic heart failure: A systematic literature review. Int J Cardiol. 2015;184: 728-735

43. Böhm M, Lloyd SM, Ford I, et al. Non-adherence to ivabradine and placebo and outcomes in chronic heart failure: an analysis from SHIFT. Eur J Heart Fail. 2016;18(6):672-683.

44. Laufs U, Rettig-Ewen V, Böhm M. Strategies to improve drug adherence. Eur Heart J. 2011;32(3):264-268.

45. Bermingham M, Hayden J, Dawkins I, et al. Prospective analysis of LDL-C goal achievement and self-reported medication adherence among statin users in primary care. Clin Ther. 2011;33(9):1180-1189.

46. Castellano JM, Sanz G, Peñalvo JL, et al. A polypill strategy to improve adherence: results from the FOCUS project. J Am Coll Cardiol. 2014; 64(20):2071-2082.
47. Gwadry-Sridhar FH, Manias E, Lal L, et al. Impact of interventions on medication adherence and blood pressure control in patients with essential hypertension: a systematic review by the ISPOR medication adherence and persistence special interest group. Value Health. 2013; 16(5):863-871.

48. Gupta AK, Arshad S, Poulter NR. Compliance, safety, and effectiveness of fixed-dose combinations of antihypertensive agents: a meta-analysis. Hypertension. 2010;55(2):399-407.

49. Krousel-Wood M, Joyce C, Holt E, et al. Predictors of decline in medication adherence: results from the cohort study of medication adherence among older adults. Hypertension. 2011;58(5):804-810.

50. Bauer LK, Caro MA, Beach SR, et al. Effects of depression and anxiety improvement on adherence to medication and health behaviors in recently hospitalized cardiac patients. Am J Cardiol. 2012;109(9) 1266-1271.

\section{Publish your work in this journal}

Patient Preference and Adherence is an international, peer-reviewed, open access journal that focuses on the growing importance of patient preference and adherence throughout the therapeutic continuum. Patien satisfaction, acceptability, quality of life, compliance, persistence and their role in developing new therapeutic modalities and compounds to optimize clinical outcomes for existing disease states are major areas of interest for the journal. This journal has been accepted for indexing on PubMed Central. The manuscript management system is completely online and includes a very quick and fair peer-review system, which is all easy to use. Visit http://www. dovepress.com/testimonials.php to read real quotes from published authors. 